\title{
USO DE IMAGENS DE ALTA RESOLUÇÃO PARA AVALIAÇÃO DE ÁREAS VERDES NA CIDADE DE SÃO PAULO, BRASIL
}

\author{
Juliana Amorim da Costa ${ }^{1}$, Demóstenes Ferreira da Silva Filho ${ }^{2}$, Jefferson Lordello Polizel ${ }^{3}$
}

(recebido em 13.09.2011 e aceito para publicação em 15.03.2012)

\section{RESUMO}

A presença de áreas verdes nas cidades traz inúmeras melhorias a estas e melhora a qualidade de vida do cidadão, exercendo funções estéticas, de lazer e educacional. O crescimento das cidades brasileiras não foi acompanhado por um planejamento urbano que reservasse espaços para o verde, como é o caso da cidade de São Paulo, uma metrópole que possui problemas sociais e ambientais. As ferramentas de geoprocessamento e sensoriamento remoto vêm sendo utilizadas com sucesso para analisar o tecido urbano e, em especial, a arborização presente na cidade. Assim, fez-se uso de imagens de alta resolução, dos satélites Ikonos e Quickbird, dos anos de 2002, 2004, 2006 e 2008, para avaliar quanto ao quesito arborização três regiões da cidade de São Paulo: subprefeitura da Mooca, subprefeitura da Sé e subprefeitura de Pinheiros. Para isto foram aplicados diferentes métodos de obtenção de dados físicos do tecido urbano, por meio de técnicas de classificação de imagens de alta resolução, juntamente com a aplicação do Índice de Floresta Urbana. O método de classificação que demonstrou ser o mais adequado para este trabalho foi o Stepwise Linear e a região que obteve melhor índice de floresta urbana foi a da subprefeitura de Pinheiros.

Palavras-chave: Sensoriamento remoto; Classificação automática; Arborização urbana; Índice de floresta urbana.

\footnotetext{
${ }^{1}$ Mestre em Ciências na Área de Recursos Florestais e Gestora Ambiental pela Escola Superior de Agricultura "Luiz de Queiroz", Universidade de São Paulo, Piracicaba, SP - juliana.costa@usp.br.

2 Professor Doutor, Departamento de Ciências Florestais, Escola Superior de Agricultura "Luiz de Queiroz" Universidade de São Paulo, Piracicaba, SP - dfilho@usp.br

3 Técnico do Laboratório de Métodos Quantitativos, Departamento de Ciências Florestais, Escola Superior de Agricultura "Luiz de Queiroz" - Universidade de São Paulo, Piracicaba, SP - jlpolize@esalq.usp.br
} 


\section{USE OF HIGH-RESOLUTION IMAGES TO EVALUATE GREEN AREAS IN THE CITY OF SAO PAULO}

\section{ABSTRACT}

The presence of green areas in the cities brings uncountable improvements to them and also makes better citizen's life, with esthetical, leisure and educational functions. The Brazilian cities growth was not supported by an urban planning that could have reserved green areas, as it is in São Paulo city, a metropolis which has social and environment problems. Geoprocessing and remote sensing have been used successfully to evaluate urban area and, specially, urban forestry presence. Thus, it was used high definition images, Ikonos and Quickbird satellites, the years 2002, 2004, 2006 e 2008, to evaluate the item urban forestry in three zones of São Paulo, using the concept of greenways: Mooca's administrative region, Sé's administrative region and Pinheiros's administrative region. For this, it was evaluated many methods to obtain physical data from São Paulo city urban area, through highresolution image classification techniques and with the implementation of the Urban Forest Index. The method Stepwise Linear proved to be most appropriate for this research and the Pinheiros's administrative region had the best value for Urban Forest Index.

Keywords: Remote sensing; Automatic classification; Urban forestry; Urban forest index

\section{INTRODUÇÃO}

A cidade de São Paulo é uma das maiores cidades mundiais e concentra mais de dez milhões de pessoas (INSTITUTO BRASILEIRO DE GEOGRAFIA E ESTATÍSTICA, IBGE, 2007). Entretanto sua urbanização não foi alvo de uma política específica que guiasse sua rápida expansão (BOLAFFI, 1992). E nesta urbanização não planejada, percebe-se que foram reservados poucos espaços vazios que pudessem servir como lazer e preservação ambiental (ALVAREZ; PENTEADO, 2006).

Como exemplos do reflexo dessa ausência de políticas públicas adequadas para a cidade de São Paulo, podem-se citar problemas ocasionados tanto na esfera econômica,

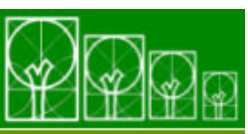

$\mathbf{S} \cdot \mathbf{B} \cdot \mathbf{A} \cdot \mathbf{U}$ Soc. Bras. de Arborização Urbana

REVSBAU, Piracicaba - SP, v.7, n.1, p. 159-181, 2012 
quanto nas esferas social e ambiental, resultando na degradação da qualidade de vida da população, por meio de altos níveis de poluição, grande quantidade de resíduos, transformação de áreas verdes em áreas impermeabilizadas, altos níveis de violência e segregação social, comprometendo a saúde física e mental desta população, que já não convive mais no espaço de rua, visto como um espaço de criminalidade (BRAGA, 1999; PILOTTO, 2003).

E a implantação de áreas verdes na cidade contribui com a amenização de diversos problemas, como ilhas de calor, enchentes e doenças respiratórias na população, do mesmo modo que contribui com a estética da cidade, com o lazer e a educação. Desta maneira fazse necessário a avaliação destas áreas para um planejamento adequado da arborização urbana.

Técnicas de sensoriamento remoto e geoprocessamento são importantes para se conseguir informações referentes à visualização e quantificação da arborização urbana e sua estrutura, permitindo uma avaliação periódica das áreas, constituindo-se de um método eficiente e econômico, possibilitando uma visão total das áreas estudadas (SILVA FILHO, 2004).

Dentro do sensoriamento remoto têm-se as imagens de alta resolução. Neste item enquadra-se, por exemplo, as imagens dos satélites IKONOS e Quickbird. O satélite IKONOS, é operado pela empresa Space Imaging, registra a radiação eletromagnética proveniente dos alvos em várias faixas espectrais, possuindo resolução espacial de $1 \mathrm{~m} x$ $1 \mathrm{~m}$ de lado de pixel. Já o satélite Quickbird é desenvolvido pela DigitalGlobe, caracteriza-se por ser de alta precisão (QUICKBIRD, 2008). Oferece $0,61 \mathrm{~m} \times 0,61 \mathrm{~m}$ de lado de pixel para as imagens pancromáticas e $2,8 \mathrm{~m} \times 2,8 \mathrm{~m}$ de lado de pixel para as imagens multiespectrais.

São necessários processamentos em imagens de satélite para a extração de alvos urbanos das imagens de maneira satisfatória. Dentro destes processamentos têm-se, como exemplo, processos de fusão e classificação automática de imagens.

A fusão de imagens permite manter a resolução espectral obtida com uma imagem multiespectral com melhora de sua resolução espacial a partir de sua imagem pancromática (CENTENO; RIBEIRO, 2007). Para este processo existem diferentes métodos.

Pinho et al (2005) apresentam três tipos de fusão realizados em imagens Quickbird: as que utilizam um modelo de domínio espacial, as de domínio espectral e aquelas que trabalham com operações algébricas. Este autor destacou a técnica conhecida como Brovey, a qual obteve como resultado imagens com uma variação de cores significativa, variando do verde brilhante ao azul escuro e diferença de tonalidade para áreas vegetadas. 
Ele opera por meio de um modelo algébrico com funções aritméticas pixel a pixel, realizando uma normalização de cores.

O processo de classificação é realizado para se obter informações da imagem que garantam uma análise satisfatória do território. Existem variados tipos de classificação automática, desde aquelas que necessitam de maior intervenção do usuário e a necessidade de coleta de amostras de pixels que constituem o treinamento, as classificações supervisionadas e aquelas que apenas necessitam da definição de alguns parâmetros, as classificações não supervisionadas.

Dentro das classificações supervisionadas é possível citar os métodos de Máxima Verossimilhança (Maximum Likelihood) e Stepwise Linear. O método de Máxima Verossimilhança avalia quantitativamente a variância e a covariância dos padrões de respostas espectrais das amostras de pixels coletadas da imagem, assumindo que possuem uma distribuição normal e também faz uso de estatísticas de probabilidade para identificar qual a classe que determinado pixel pertence (LILLESAND et al., 2004). Enquanto que o método Stepwise Linear faz uso de análise linear discriminante para encontrar a classe referente a determinado conjunto de pixels (MICROIMAGES, 2006).

Para as classificações automáticas não supervisionadas tem-se, entre outros, os métodos K-Means e ISODATA (Iterative Self-Organizing Data Analysis). O método K-Means agrupa os pixels na quantidade de classes definida pelo usuário, calculando o centróide de cada classe para poder distribuir esses pixels. O método ISODATA também utiliza o valor do centro de cada classe para agrupar os pixels, além de aplicar técnicas de fusão, exclusão e divisão para a classificação (LILLESAND et al., 2004).

Com a utilização dessas técnicas de geoprocessamento e sensoriamento remoto foram avaliadas as áreas verdes de três regiões da cidade de São Paulo.

Este trabalho teve como objetivo geral avaliar a arborização urbana em três subprefeituras da cidade de São Paulo: Mooca, Sé e Pinheiros. E como objetivos específicos:

(i) Análise de diferentes modelos de obtenção de dados físicos do tecido urbano da cidade de São Paulo por meio de sensoriamento remoto e sistemas de informação geográfica para a definição do melhor método para eleição de áreas prioritárias;

(ii) Análise de imagens de satélite de diferentes épocas da cidade, para verificação das transformações das áreas verdes nas três regionais em estudo;

(iii) Aplicação do Índice de Floresta Urbana, proposto por Silva Filho et al. (2005).

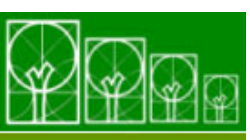

$\mathbf{S} \cdot \mathbf{B} \cdot \mathbf{A} \cdot \mathbf{U}$ Soc. Bras. de Arborização Urbana 


\section{MATERIAIS E MÉTODOS}

\section{Área de estudo}

\section{O município de São Paulo}

O município de São Paulo possui extensão de $1.530 \mathrm{~km}^{2}$ de área, altitude $780 \mathrm{~m}$ acima do nível do mar, localizada na latitude de $23^{\circ} 32.0^{\prime} S$ e na longitude de $46^{\circ} 37.0^{\prime} \mathrm{W}$, no estado de São Paulo, região Sudeste, Brasil (CIDADE DE SÃO PAULO, 2008), com uma população de 10.886.518 habitantes (IBGE, 2007). Caracterizado por clima subtropical (tipo Cwa segundo Köppenn), com temperaturas médias anuais de $19^{\circ} \mathrm{C}$.

Dentro desta região foram estudadas e analisadas as subprefeituras da Mooca, da Sé, e de Pinheiros, que podem ser observadas na Figura 1.

Figura 1. Área de estudo

Figure 1. Study área

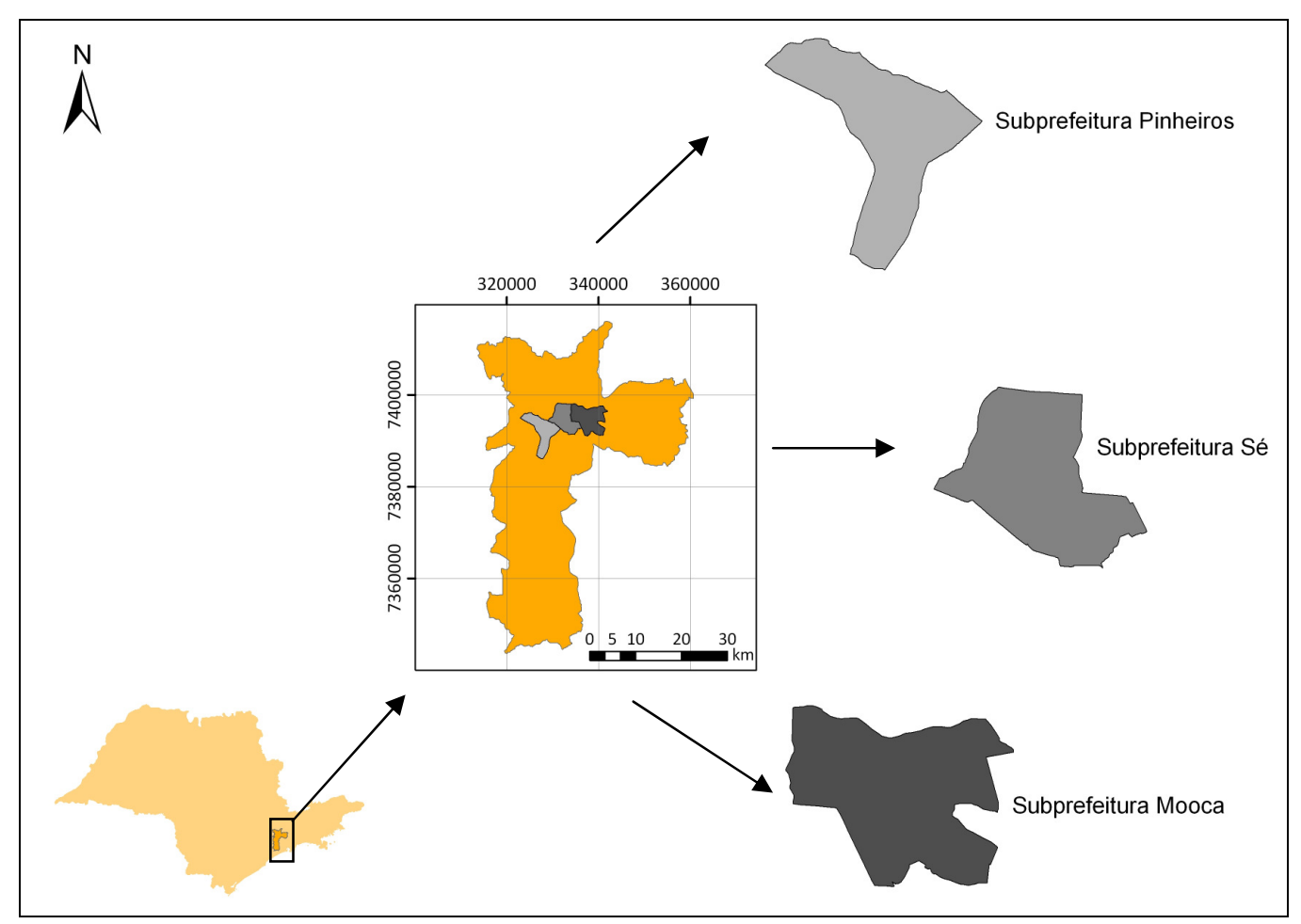

A subprefeitura da Mooca possui uma área de $35,2 \mathrm{~km}^{2}$ e se encontra na região leste da cidade de São Paulo. Fazem parte desta subprefeitura os seguintes bairros: Mooca, Água Rasa, Belém, Tatuapé, Pari e Brás, com uma população de 308.161 habitantes, de 
acordo com o censo demográfico do IBGE de 2000 (PORTAL DA PREFEITURA DA CIDADE DE SÃO PAULO, 2009), densidade demográfica de 8.754,57 hab/km².

Localizada no centro da área de estudo, a subprefeitura da Sé engloba uma área de $26,2 \mathrm{~km}^{2}$. Esta regional é responsável pelos bairros Bom Retiro, Santa Cecília, República, Sé, Consolação, Bela Vista, Liberdade e Cambuci, e possui uma população de 373.914 habitantes, de acordo com o censo demográfico do IBGE de 2000 (PORTAL DA PREFEITURA DA CIDADE DE SÃO PAULO, 2009), densidade demográfica de 14.271,53 $\mathrm{hab} / \mathrm{km}^{2}$.

A subprefeitura de Pinheiros, localizada na região oeste da cidade, possui uma área de $31,7 \mathrm{~km}^{2}$. Os bairros que fazem parte da mesma são: Alto de Pinheiros, Pinheiros, Itaim Bibi e Jardim Paulista. Possui 272.574 habitantes, de acordo com o censo demográfico do IBGE de 2000 (PORTAL DA PREFEITURA DA CIDADE DE SÃO PAULO, 2009), densidade demográfica de 8.598,55 hab/km².

Para o trabalho foi utilizado o Centro de Métodos Quantitativos, do Departamento de Ciências Florestais da Escola Superior de Agricultura Luiz de Queiroz (ESALQ), Universidade de São Paulo (USP), localizado na cidade de Piracicaba, São Paulo, Brasil. Também foram realizadas visitas de campo nas regiões estudadas.

\section{Processamento das imagens}

Nesta etapa fez-se uso de imagens do satélite IKONOS do ano de 2002, ortorretificadas a partir do modelo digital de elevação, entregues em quatro bandas espectrais, no domínio do visível (RGB), com as bandas vermelha (Red), verde (Green) e azul (Blue), e do infravermelho próximo, com resolução espacial de 1 metro e resolução radiométrica de 11 bits (MOREIRA, 2005).

Estas imagens foram adquiridas pelo Instituto Geológico, pertencente à Secretaria de Meio Ambiente do Estado de São Paulo, no ano de 2003, por meio da FUNCATE (Fundação de Ciência, Aplicações e Tecnologias Espaciais). Cabe ressaltar que estas imagens estão mosaicadas, portanto, não foi possível aplicar correções radiométricas nas mesmas.

O imageamento original foi executado pelo satélite IKONOS, operado pela Space Imaging, em outubro de 2002 e complementado por novas aquisições de imagens em 2003.

Também foram utilizadas imagens do satélite Quickbird desenvolvido pela Digital Globe. Adquiriram-se imagens das subprefeituras da Mooca, da Sé e de Pinheiros para os anos de 2004, 2006 e 2008. Entretanto, para o ano de 2004 a imagem disponível em

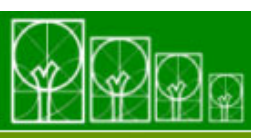

$\mathbf{S} \cdot \mathbf{B} \cdot \mathbf{A} \cdot \mathbf{U}$ Soc. Bras. de Arborização Urbana 
catálogo não abrangia toda a região da subprefeitura de Pinheiros. E para o ano de 2006, a imagem disponível em catálogo não abrangia toda a área da subprefeitura da Sé, e não havia imagem disponível para a região da subprefeitura de Pinheiros. As imagens estão no sistema de coordenadas UTM (Universal Transverse Mercator), zona 23, datum WGS-84.

Para analisar as imagens obtidas foram utilizados os programas de geoprocessamento ArcGIS 9.2, desenvolvido pela ESRI e TNTmips 2009, desenvolvido pela Microimages.

\section{Fusão das imagens Quickbird}

As imagens Quickbird passaram por processo de fusão da imagem pancromática com a multiespectral, por meio de técnicas de geoprocessamento do programa TNTmips 2009, sendo utilizado o método Brovey para tal processamento. Testes foram realizados para saber qual combinação de bandas durante a fusão seria a mais interessante para a análise da cobertura arbórea da região em estudo.

Os testes de fusão foram validados por meio dos testes de classificação automática supervisionada, os quais serão explicados nos próximos itens. As imagens utilizadas para os testes foram as Quickbird do ano de 2008.

As seguintes combinações de bandas da imagem foram analisadas:

(i) Teste 1: utilização das bandas do azul, vermelho e infravermelho próximo, mais a banda pancromática;

(ii) Teste 2: utilização das quatro bandas multiespectrais mais a pancromática;

(iii) Teste 3: utilização das bandas verde, vermelha e do infravermelho próximo, mais a banda pancromática.

\section{Classificação automática de imagens de alta resolução}

As classificações testadas foram a classificação supervisionada, dentro desta o método de Máxima Verossimilhança e o método Stepwise Linear. Estes foram avaliados por análise multivariada discreta conhecida como estatística Kappa e interpretação visual e comparativa; e a classificação não supervisionada, fazendo uso dos métodos ISODATA e KMeans. Estes foram avaliados por meio de comparação com as imagens originais e com os outros métodos de classificação. O índice Kappa é obtido pelas fórmulas 1, 2 e 3 (LANDIS; $\mathrm{KOCH}, 1977)$. 


$$
\begin{gathered}
K=\frac{P o-P c}{1-P c} \\
P o=\frac{\sum_{i=1}^{M} n_{i j}}{N} \\
P c=\frac{\sum_{i=1}^{M} n_{i+} n_{j+}}{N^{2}}
\end{gathered}
$$

Onde: $\mathrm{Po}=$ proporção de concordância observada; $\mathrm{Pc}=$ proporção de concordância esperada ao acaso; $\mathrm{M}=$ número de classes presentes na matriz de erro; $\mathrm{n}_{\mathrm{ij}}=$ número de observações na linha $i$ e coluna $j ; n_{i_{+}}$e $n_{j_{+}}=$totais marginais da linha $i$ e da coluna $j$, respectivamente; e $\mathrm{N}=$ número total de unidades amostrais contempladas pela matriz.

De acordo com Landis e Koch (1977), tem-se a seguinte interpretação desse índice (Tabela 1):

Tabela 1 - Interpretação do índice Kappa

Table 1 - Interpretation of Kappa Index

\begin{tabular}{cc}
\hline Valores de Kappa (\%) & Exatidão da Classificação \\
\hline 0 & Nula \\
$0-20$ & Pobre \\
$21-40$ & Fraca \\
$41-60$ & Moderada \\
$61-80$ & Boa \\
$81-100$ & Ótima \\
\hline
\end{tabular}

Para a validação dos testes de classificação supervisionada também foi analisado o dendrograma de cada imagem classificada.

Após realizada a classificação foi aplicado um filtro na imagem classificada, chamado Hole-Filling, que substitui a classe de um pixel, por aquela em que se encontra a maioria das células vizinhas, removendo aquelas que se encontram numa classe isolada. É possível escolher qual o tamanho da área a ser filtrada e neste trabalho escolheu-se o tamanho $3 \times 3$.

As classes utilizadas foram: copa arbórea, relvado, asfalto, piso cimento, telha cerâmica, telha cinza, telha escura, telha metálica, sombra, solo exposto e outros. Estas são as classes utilizadas para a caracterização do uso das terras em trabalhos já realizados pelo Laboratório de Silvicultura Urbana da ESALQ/USP.

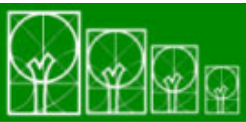




\section{Cálculo do Índice de Floresta Urbana}

Para analisar a cobertura arbórea nas regiões de estudo foi calculado o Índice de Floresta Urbana (IFU) proposto por Silva Filho et al. (2005). Este é um indicador para valorização de espaços arborizados que relaciona esses espaços com os outros elementos presentes na paisagem urbana. É obtido por meio da fórmula 4:

$$
I F U=P A I+P A C
$$

Sendo que os índices PAl (proporção entre Espaço Arborizado e Espaço Livre Impermeável) e PAC (proporção entre Espaço Arborizado e Espaço Construído) são obtidos por meio das fórmulas 5 e 6 :

$$
\begin{aligned}
& P A I=\frac{E L A}{(E L A+E L I)} \\
& P A C=\frac{E L A}{(E L A+E C)}
\end{aligned}
$$

Onde: ELA representa o espaço livre arborizado (classe copa de árvore); ELI representa o espaço livre impermeabilizado (classes asfalto e piso cimento); EC é o espaço construído (classes telha cerâmica, telha metálica, telha escura e telha cinza).

O IFU varia entre os valores zero e dois, sendo que quanto maior o valor do índice, maior é a porcentagem de cobertura arbórea em relação aos demais elementos do tecido urbano (SILVA FILHO et al., 2005).

\section{RESULTADOS E DISCUSSÃO}

\section{Fusão de imagens Quickbird}

Realizou-se a fusão das bandas mutiespectrais de resolução espacial de 2,8 metros da imagem Quickbird, em diferentes combinações, com a banda pancromática, de resolução espacial de 0,61 metros, no programa TNTmips 2009, que possui uma função específica para a fusão de imagens Quickbird, sendo aplicado o método Brovey. 
Este método combina bandas multiespectrais de baixa resolução espacial, representada como $\mathrm{R}$ (Vermelho), $\mathrm{G}$ (Verde) e $\mathrm{B}$ (Azul), de uma imagem com a banda pancromática, de alta resolução espacial. Este método faz uso de técnicas aritméticas de soma e multiplicação (VRABEL, 1996).

A diferente combinação das bandas das imagens multiespectrais fornece diferentes respostas espectrais, já que são diferentes combinações de comprimentos de onda para os mesmos alvos de uma determinada imagem.

A partir da classificação automática supervisionada realizou-se uma análise visual e análise do dendrograma obtido pelo programa TNTmips 2009. Constatou-se que as diferentes combinações de bandas multiespectrais utilizadas para a fusão da imagem influenciam nos resultados de classificação automática, principalmente no que tange à porcentagem de cada classe encontrada na imagem. Para exemplificar, segue a Tabela 2 que mostra a porcentagem de classes da subprefeitura da Mooca com os resultados para o classificador de Máxima Verossimilhança.

Tabela 2 - Porcentagem das classes para a subprefeitura da Mooca utilizando o método de classificação de Máxima Verossimilhança

Table 2 - Percentage of classes for the Mooca's administrative region using the method of Maximum likelihood Classification

\begin{tabular}{lccc}
\hline Classe & Teste 1 (\%) & Teste 2 (\%) & Teste 3 (\%) \\
\hline Piscina & 0,06 & 0,08 & 0,04 \\
Copa de Árvore & 5,45 & 7,39 & 9,16 \\
Solo Exposto & 2,57 & 2,54 & 3,78 \\
Asfalto & 14,10 & 14,95 & 14,40 \\
Relvado & 7,06 & 2,40 & 10,07 \\
Telha Escura & 33,85 & 24,02 & 30,64 \\
Telha Cerâmica & 3,72 & 3,39 & 4,05 \\
Sombra & 12,81 & 15,85 & 11,96 \\
Telha Metálica & 0,25 & 0,23 & 0,60 \\
Telha Cinza & 3,84 & 3,79 & 2,40 \\
Lago/Rio & 2,17 & 0,01 & 0,42 \\
Piso Cimento & 11,71 & 11,66 & 5,90 \\
Outros & 2,41 & 13,70 & 5,58 \\
\hline
\end{tabular}


Observa-se na classe "copa de árvore", a de maior interesse nesse estudo, a existência de grande diferença entre os valores de porcentagem encontrados para cada tipo de combinação de bandas. Isso evidencia a necessidade de testes para saber qual a melhor combinação a ser utilizada para se extrair dados de uma imagem de satélite.

Com a diferente resposta espectral dos alvos de estudo, dependendo da fusão realizada, verifica-se a diferença na quantidade de pixels encontrados para cada classe.

Além disso, com a análise do dendrograma é possível avaliar o grau de separabilidade dos alvos. Desta maneira, a combinação mais adequada de bandas, foi aquela que mostrou menor separabilidade entre os alvos que possuem material semelhante em sua constituição, como é o caso de copa de árvore e relvado, mas que apresentam alta separabilidade entre os diferentes alvos.

Assim, constatou-se que a fusão mais adequada para extração de dados de cobertura arbórea foi aquela com a combinação de bandas azul, vermelha e do infravermelho próximo fusionadas com a banda pancromática (Teste 1).

\section{Obtenção de dados físicos a partir de imagens de alta resolução}

\section{Classificadores não supervisionados}

O usuário deve fornecer ao algoritmo o número de classes espectrais prováveis, a distância mínima desejada entre os valores dos níveis digitais de duas classes e o número de iterações realizadas. Este método faz o agrupamento de pixels homogêneos em $n$ classes espectrais (MOREIRA, 2005). Limitou-se o algoritmo em 13 classes e 20 iterações.

Os testes com o classificador K-Means demonstraram não ser este apropriado para extração de dados de elementos da paisagem urbana numa imagem de alta resolução, seja ela IKONOS ou Quickbird.

Verificou-se que o classificador faz confusão entre classes. Há áreas classificadas como "solo exposto" que na realidade são "telhas cinza" ou "piso cimento". Além de "telhas cerâmicas" que foram classificadas como "relvado". "Telha escura", "piso cimento" e "asfalto" foram classes não diferenciadas pelo algoritmo.

Com a análise dos dendrogramas, observou-se o grau de separabilidade entre as classes. Constatou-se que elementos que deveriam estar com um grau de separabilidade menor, por serem constituídos de materiais de resposta espectral semelhante, estavam distantes e próximos a elementos de resposta espectral totalmente distinta. 
A classe "copa de árvore", seguindo o mesmo raciocínio, deveria estar próxima à classe "relvado", porém não apresenta esse comportamento. Aproxima-se de elementos que deveriam ter um grau de separabilidade maior, já que suas respostas espectrais são muito diferentes, como com a classe "cimento/concreto".

O método de classificação não supervisionado ISODATA é similar ao K-Means, mas incorpora critérios de divisão, combinação e descarte para as classes a fim de obter um número satisfatório de classes de saída. Baseia-se na análise de agrupamentos onde são identificados pixels com características similares (MICROIMAGES, 2006). Foram definidos no programa o limite de 13 classes e 20 iterações.

Os testes com o classificador ISODATA demonstraram não ser este apropriado para extração de dados de elementos da paisagem urbana numa imagem de alta resolução, seja ela IKONOS ou Quickbird.

Verificou-se que o mesmo não consegue separar alguns elementos, como "telha cerâmica" e "piscina" e não distingue outros alvos. Observou-se também uma forte mistura entre os elementos "piso cimento", "telha escura" e "asfalto", e também no que se refere à "copa de árvore" e "relvado".

Esta confusão entre classes pelo método ISODATA também foi identificada por Valerio et al. (2008). O autor gerou mapas temáticos de uso do solo e de qualidade da água no Reservatório do Rio Manso em Cuiabá, MT. E comparou o classificador ISODATA com um classificador supervsionado de Máxima Verossimilhança, utilizando imagens Landsat TM. Este último classificador apresentou melhor correlação entre a imagem original e a imagem classificada. No mapa de qualidade da água que possuía menos elementos para serem classificados do que o mapa de uso do solo, o método ISODATA apresentou menor confusão entre as classes.

Os dendrogramas dessas imagens, como no caso do classificador K-Means, demonstraram maior separabilidade entre classes de semelhante resposta espectral, do que entre classes com diferente resposta espectral.

\section{Classificadores supervisionados}

Classificadores automáticos supervisionados são aqueles que exigem a confecção de um treinamento para serem aplicados. O treinamento deve coletar amostras distribuídas por toda imagem para que seja representativo.

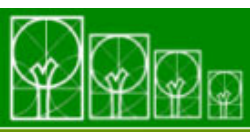

$\mathbf{S} \cdot \mathbf{B} \cdot \mathbf{A} \cdot \mathbf{U}$ Soc. Bras. de Arborização Urbana 
Os resultados obtidos com o classificador de Máxima Verossimilhança demonstraram que o mesmo superestima a quantidade de cobertura arbórea, já que classifica áreas de relvado e algumas áreas de solo exposto como pertencentes à classe copa de árvore.

Ribeiro e Centeno (2001) também observaram esse fato quando classificaram imagens Landsat TM. Os autores identificaram que classes espectralmente próximas não eram separadas no processo de classificação, exemplificando com as classes "cultura" e "pastagem". E concluem dizendo que uma quantidade maior de amostras no treinamento pode gerar uma melhora do resultado.

Ouma e Tateishi (2008) em um trabalho sobre extração de dados de cobertura arbórea em áreas urbanas, utilizando imagens Quickbird, também concluíram que o classificador de máxima verossimilhança superestimou a quantidade de árvores urbanas. Os autores observaram que este algoritmo possui dificuldade em distinguir estatisticamente pixels pertencentes às classes relvado, arbusto, árvores e sombra.

O método Stepwise Linear também apresentou certa confusão entre classes, como entre "relvado" e "copa de árvore". Entretanto, por meio de análise visual dos testes realizados, verificou-se que essa confusão é menor quando comparada com as classificações realizadas com o método de Máxima Verossimilhança.

Weber, Petropoulou e Hirsch (2005) estudaram o desenvolvimento da área metropolitana de Atenas, na Grécia, utilizando técnicas de geoprocessamento. O autor realizou uma classificação supervisionada com o método Stepwise Linear em imagens do satélite SPOT e verificou que este classificador conseguiu diferenciar os alvos presentes na paisagem urbana, de acordo com características como presença/ausência de vegetação e presença/ausência de áreas construídas. Dessa maneira demonstrou ser um classificador adequado para áreas urbanas.

Os dendrogramas analisados mostram uma separabilidade menor entre alvos de semelhante resposta espectral, como "copa de árvore" e "relvado" e separabilidade maior entre aqueles com diferente resposta espectral. O classificador Stepwise Linear mostra-se mais fiel à verdade terrestre quando se trata da classe copa de árvore, alvo de interesse desse estudo.

Desta maneira, para a extração de dados de cobertura arbórea das regiões de estudo foi aplicado o método Stepwise Linear para a classificação das imagens de alta resolução.

Ressalta-se que atualmente novas técnicas de classificação também são utilizadas para imagens de alta resolução, como a classificação orientada a objeto, a qual utiliza diversos outros parâmetros para realizar a classificação. Destaca-se então a importância de 
trabalhos que comparem essas técnicas e assim ter o conhecimento de qual apresenta melhores resultados para cada tipo de estudo.

\section{Quantificação de cobertura arbórea em áreas urbanas}

Foram utilizadas imagens de diferentes anos das áreas de estudo para quantificar a cobertura arbórea da região. Cabe ressaltar que as imagens foram coletadas em meses diferentes. As imagens Quickbird são de agosto de 2004, novembro de 2006 e junho de 2008. Portanto são de diferentes épocas do ano, em que as copas podem estar mais ou menos vigorosas, sendo assim influenciam na análise quantitativa da classe "cobertura arbórea" e essa informação deve ser cuidadosamente considerada.

\section{Subprefeitura da Mooca}

Foi realizado um estudo de campo para caracterizar a região. Observou-se que esta é marcada, sobretudo, por áreas comerciais, apresentando, assim, grande fluxo de pessoas durante a semana e aos sábados, o que traz certo grau de degradação à região no que diz respeito, principalmente, à lixo jogado nas ruas e falta de cuidado com os equipamentos públicos. Não foi observada presença acentuada de arborização no viário. A Tabela 3 contém as porcentagens dos elementos presentes na região

Tabela 3 - Cobertura do solo da região da subprefeitura da Mooca nos anos de 2002, 2004, 2006 e 2008

Table 3 - Land cover Mooca's administrative region in 2002, 2004, 2006 and 2008

\begin{tabular}{lllll}
\hline Classe & $2002(\%)$ & $2004(\%)$ & $2006(\%)$ & $2008(\%)$ \\
\hline Piscina & 0,06 & 0,03 & 0,04 & 0,04 \\
Copa de Árvore & 2,78 & 1,98 & 4,35 & 3,13 \\
Solo Exposto & 9,04 & 2,45 & 4,69 & 4,51 \\
Asfalto & 20,60 & 29,53 & 17,50 & 20,10 \\
Relvado & 1,18 & 1,50 & 1,89 & 3,55 \\
Telha Escura & 19,31 & 21,78 & 37,53 & 27,41 \\
Telha Cerâmica & 12,88 & 1,42 & 6,93 & 2,52 \\
Sombra & 14,44 & 19,97 & 6,66 & 26,33 \\
Telha Metálica & 1,02 & 0,69 & 3,66 & 0,53
\end{tabular}




\begin{tabular}{lllll} 
Telha Cinza & 7,73 & 2,57 & 3,27 & 3,85 \\
Lago/Rio & - & 0,60 & 0,42 & 0,15 \\
Piso Cimento & 6,72 & 7,51 & 12,62 & 6,67 \\
Outros & 4,25 & 9,99 & 0,45 & 1,22 \\
\hline
\end{tabular}

As classificações foram avaliadas por meio da estatística Kappa (Figura 2). Na matriz de erro, além do índice Kappa, devem ser analisados os valores apresentados por cada classe na matriz de erro, isto é, o número de pixels representativos de cada classe. $\mathrm{Na}$ matriz apresentada estes números são observados em destaque na cor vermelha. Para que o resultado da classificação seja considerado satisfatório, estes números devem estar dispostos como uma "escada", representando que o maior número de pixels amostrados naquela classe realmente pertence à mesma. Todas as matrizes de erro apresentadas, referentes às classificações realizadas, apresentaram esse comportamento.

Todas as classificações se enquadraram como ótima, de acordo com a estatística Kappa, como pode ser observado na Tabela 4 (LANDIS; KOCH, 1977).

Figura 2 - Matriz de erro de classificação da imagem IKONOS de 2002 para a subprefeitura da Mooca

Figure 2 - IKONOS classified image Error Matrix to Mooca's administrative region in 2002

\begin{tabular}{|c|c|c|c|c|c|c|c|c|c|c|c|c|c|c|}
\hline \multicolumn{15}{|c|}{ Ground Truth Data } \\
\hline Chang & Piscina & Copa de & Relvado & Telha az: & Solo exp & Asfalto & 5onbra & Telha ne & Telha ce & Piso cin & Telha es 1 & Tolha cil & Total & fiecuract \\
\hline I Piscina & 1730 & 0 & 0 & 0 & 0 & 0 & 0 & 0 & 0 & 0 & 0 & 0 & 1730 & $100.00 \%$ \\
\hline Copa do & 0 & 1650 & 5 & 0 & 0 & 0 & 0 & 0 & 4) & 0 & 0 & 0 & 1659 & $99.46 \mathrm{x}$ \\
\hline Roluado & 0 & 141 & 2555 & 0 & 0 & 0 & 0 & 0 & 20 & 0 & 0 & 0 & 2724 & 93.600 \\
\hline Iolha az & 1 & 1 & 0 & 498 & 0 & 2 & 0 & 0 & 0 & 2 & 11 & 4 & 519 & $95.95 x$ \\
\hline 5olo axp & 0 & 0 & 0 & 0 & 1741 & 1 & 0 & 0 & 153 & 46 & 2 & 0 & 1943 & $69.60 \%$ \\
\hline cisfalto & 0 & 0 & 0 & 0 & 0 & 1384 & 0 & 0 & 0 & 5 & 497 & 76 & 1962 & 70.54 \\
\hline a Sombra & 0 & 0 & 0 & 0 & 0 & 0 & 3873 & 0 & 0 & 0 & 183 & 0 & 4056 & 95.497 \\
\hline telha ne & 0 & 0 & 0 & 0 & 0 & 0 & 0 & 1503 & 0 & 5 & 0 & 0 & 1500 & $99.67 x^{\prime}$ \\
\hline Telha ce & 0 & 26 & 166 & 0 & 4 & 0 & 0 & 0 & 556 & 3 & 0 & 0 & 755 & 7.64 \\
\hline n Piso cin & 0 & 0 & 0 & 0 & 101 & 0 & 0 & 0 & 0 & 361 & 0 & 72 & 534 & 67.600 \\
\hline Telho os & 0 & 0 & 0 & 4 & 0 & 263 & 40 & 0 & 3 & 0 & 1863 & 0 & 2193 & 04.95\% \\
\hline Telha ci & 18 & 0 & 0 & 0 & 9 & 23 & 0 & 0 & 0 & 27 & 0 & 3277 & 3354 & $97.70 t$ \\
\hline t Total & 1749 & 1818 & 2726 & 502 & 1855 & 1693 & 3913 & 1503 & 744 & 449 & 2556 & 3429 & 22937 & \\
\hline Rigcur acy & 90.917 & 90.76 & $93.73 x$ & 99.207 & $93.85 \%$ & 81.7.74 & $90.90 \mathrm{x}$ & 100,007 & 74.737 & $0,0.406$ & 72_698: & $95.57 /$ & & \\
\hline
\end{tabular}


Tabela 4 - Valores de Kappa e Acurácia para as classificações da subprefeitura da Mooca Table 4 - Kappa and Accuracy values to classified images from Mooca's administrative region

\begin{tabular}{lllll}
\hline Avaliação & $2002(\%)$ & $2004(\%)$ & $2006(\%)$ & $2008(\%)$ \\
\hline Kappa & 90,49 & 85,61 & 93,47 & 85,63 \\
Acurácia & 91,52 & 87,10 & 94,37 & 87,06 \\
\hline
\end{tabular}

As classes foram distribuídas nos seguintes grupos para o cálculo do IFU (SILVA FILHO et al., 2005):

(i) Espaços livres vegetados: copa de árvore e relvado;

(ii) Espaços livres permeáveis: solo exposto;

(iii) Espaços livres impermeáveis: piso cimento e asfalto;

(iv) Espaços construídos: telha cerâmica, telha metálica, telha escura e telha cinza;

(v) Espaços d'água: lago/rio e piscina;

(vi) Outros: sombra e outros.

Foram identificados na classe de cobertura "outros" helipontos e quadras poliesportivas. A classe de cobertura "lago/rio" foi "mascarada" (retirada da imagem para a classificação) na imagem IKONOS de 2002, pois estava sendo confundida fortemente com as outras classes. Além disso, não é uma classe quantitativamente significativa para a região.

Nuvens e sombras são fatores que prejudicam a classificação (RIBEIRO; CENTENO, 2001). Assim foi criada uma classe especial para sombra, e as nuvens foram desconsideradas pelo classificador, aplicando-se uma máscara nessas regiões.

A classe "sombra" influencia nas porcentagens de outras classes, já que varia de imagem para imagem, e esconde os elementos na mesma. Por isso pode-se observar na tabela algumas variações bruscas de porcentagem em alguns elementos. Entretanto, a principal classe de estudo, "copa de árvore", não sofreu variações repentinas ao longo dos anos.

Chegou-se aos seguintes resultados para o IFU (Tabela 5):

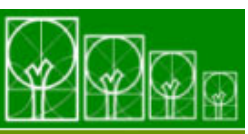


Tabela 5 - Índice de Floresta Urbana para a subprefeitura da Mooca

Table 5 - Mooca's administrative region Urban Forest Index

\begin{tabular}{cc}
\hline Período & IFU \\
\hline 2002 & 0,155945 \\
2004 & 0,120363 \\
2006 & 0,204238 \\
2008 & 0,188283 \\
\hline
\end{tabular}

A baixa presença de áreas arborizadas constatadas em campo foi confirmada pelo baixo valor do IFU para o período de estudo. O índice sofreu no geral um pequeno aumento durante os anos de 2002 a 2008, sendo que houve uma leve diminuição do ano de 2006 para o ano de 2008.

Silva Filho et al. (2005) aplicaram o IFU para nove bairros da cidade de Piracicaba/SP. O menor valor encontrado foi para o bairro Cidade Alta, com IFU de 0,445. Mesmo este sendo o menor valor, é superior àquele encontrado para a região da subprefeitura da Mooca. O que demonstra a reduzida quantidade de árvores na região, e a necessidade de políticas públicas voltadas para o aumento do verde e de educação para a valorização de espaços vegetados na cidade.

\section{Subprefeitura da Sé}

Foi realizado um estudo de campo para caracterizar a região. Aqui há um misto de áreas residenciais com áreas comerciais e até industriais. A região mais central apresentase um tanto quanto degradada, principalmente no que se refere aos equipamentos públicos. Não foi observada presença marcante de árvores no viário, apesar de possuir algumas praças arborizadas, como a Praça da República. A Tabela 6 apresenta a distribuição dos elementos presentes na subprefeitura da Sé. 
Tabela 6 - Cobertura do solo da região da subprefeitura da Sé nos anos de 2002, 2004, 2006 e 2008

Table 6 - Land cover Sé's administrative region in 2002, 2004, 2006 and 2008

\begin{tabular}{lllll}
\hline Classe & $2002(\%)$ & $2004(\%)$ & $2006(\%)^{*}$ & $2008(\%)$ \\
\hline Piscina & 0,03 & 0,04 & 0,03 & 0,03 \\
Copa de Árvore & 6,96 & 3,05 & 6,87 & 4,99 \\
Solo Exposto & 4,62 & 1,51 & 2,08 & 2,22 \\
Asfalto & 12,78 & 21,26 & 16,90 & 13,88 \\
Relvado & 0,80 & 1,47 & 1,60 & 4,51 \\
Telha Escura & 23,96 & 25,61 & 26,20 & 26,55 \\
Telha Cerâmica & 5,47 & 1,49 & 12,45 & 0,65 \\
Sombra & 10,56 & 31,29 & 9,06 & 35,26 \\
Telha Metálica & 2,40 & 0,40 & 3,42 & 0,60 \\
Telha Cinza & 3,05 & 7,96 & 3,57 & 2,62 \\
Lago/Rio & 13,31 & - & - & - \\
Piso Cimento & 12,97 & 4,63 & 14,26 & 5,85 \\
Outros & 3,10 & 1,30 & 3,56 & 2,89 \\
\hline
\end{tabular}

Todas as classificações se enquadraram como ótima, de acordo com a estatística Kappa, como pode ser observado na Tabela 7 (LANDIS; KOCH, 1977).

As classes foram distribuídas nos mesmos grupos especificados na análise da subprefeitura da Mooca. Chegou-se aos seguintes resultados para o IFU (Tabela 8):

Tabela 7 - Valores de Kappa e Acurácia para as classificações da subprefeitura da Mooca Table 7 - Kappa and Accuracy values to classified images from Sé's administrative region

\begin{tabular}{llll}
\hline Avaliação & $2002(\%)$ & $2004(\%)$ & $2008(\%)$ \\
\hline Kappa & 95,47 & 89,25 & 87,72 \\
Acurácia & 96,24 & 92,03 & 89,37 \\
\hline
\end{tabular}


Tabela 8 - Índice de Floresta Urbana para a subprefeitura da Sé

Table 8 - Sé's administrative region Urban Forest Index

\begin{tabular}{cc}
\hline Período & IFU \\
\hline 2002 & 0,379127 \\
2004 & 0,184591 \\
2008 & 0,342781 \\
\hline
\end{tabular}

A baixa presença de áreas arborizadas constatadas em campo foi confirmada pelo baixo valor do IFU para o período de estudo. O índice sofreu no geral uma diminuição de 2002 a 2004, sendo que houve um aumento do ano de 2004 para o ano de 2008.

Em comparação com o trabalho de Silva Filho et al. (2005) que aplicou o IFU para bairros da cidade de Piracicaba/SP, o menor valor encontrado para um bairro em Piracicaba $(0,445)$ ainda é maior do que os valores encontrados para a região da subprefeitura da Sé.

\section{Subprefeitura de Pinheiros}

Comparando-se com as outras regiões, foi observada uma maior quantidade de árvores no viário nessa região. Aqui predominam construções recentes e com características residenciais.

O estudo foi prejudicado por dois fatores referentes às imagens de 2004 e 2006, pois para esta subprefeitura a imagem referente ao ano de 2004 está incompleta e não há imagem em catálogo para o ano de 2006. Desta maneira foram desconsideradas do cálculo do IFU. A Tabela 9 mostra a distribuição dos elementos presentes na região.

Tabela 9 - Cobertura do solo da região da subprefeitura de Pinheiros nos anos de 2002, 2004 e 2008

Table 9 - Land cover Pinheiros's administrative region in 2002, 2004 and 2008

\begin{tabular}{llll}
\hline Classe & $2002(\%)$ & $2004(\%)^{\star}$ & $2008(\%)$ \\
\hline Piscina & 0,53 & 0,12 & 0,08 \\
Copa de Árvore & 6,89 & 6,34 & 7,93 \\
Solo Exposto & 10,45 & 2,53 & 1,68 \\
Asfalto & 13,57 & 16,07 & 20,76 \\
Relvado & 1,37 & 1,55 & 10,48
\end{tabular}




\begin{tabular}{llll} 
Telha Escura & 33,14 & 32,02 & 15,31 \\
Telha Cerâmica & 7,44 & 2,38 & 1,16 \\
Sombra & 12,98 & 27,13 & 33,10 \\
Telha Metálica & 3,46 & 0,85 & 0,71 \\
Telha Cinza & 4,13 & 1,82 & 1,76 \\
Lago/Rio & - & - & - \\
Piso Cimento & 6,03 & 7,30 & 5,81 \\
Outros & - & 1,88 & 1,20 \\
\hline
\end{tabular}

Todas as classificações se enquadraram como ótima, de acordo com a estatística Kappa, como pode ser observado na Tabela 10 (LANDIS; KOCH, 1977).

Tabela 10 - Valores de Kappa e Acurácia para as classificações da subprefeitura da Mooca Table 10 - Kappa and Accuracy values to classified images from Pinheiros's administrative region

\begin{tabular}{lll}
\hline Avaliação & $2002(\%)$ & $2008(\%)$ \\
\hline Kappa & 92,82 & 82,22 \\
Acurácia & 95,40 & 93,27 \\
\hline
\end{tabular}

As classes foram distribuídas nos mesmos grupos já especificados na análise das outras subprefeituras. Foram encontrados os seguintes valores para o IFU (Tabela 11):

Tabela 11 - Índice de Floresta Urbana para a subprefeitura de Pinheiros Table 11 - Pinheiros's administrative region Urban Forest Index

\begin{tabular}{cc}
\hline Período & IFU \\
\hline 2002 & 0,385234 \\
2008 & 0,524980 \\
\hline
\end{tabular}

A região da subprefeitura de Pinheiros foi a que apresentou maiores índices de floresta urbana. Sendo que no período entre os anos de 2002 e 2008 houve um aumento desse índice. Como constatado em campo como a região mais arborizada quando comparada às outras duas subprefeituras, os valores do IFU confirmam este dado.

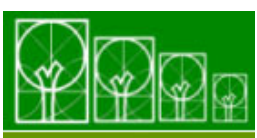

$\mathbf{S} \cdot \mathbf{B} \cdot \mathbf{A} \cdot \mathbf{U}$ Soc. Bras. de Arborização Urbana 
Em comparação com o trabalho de Silva Filho et al. (2005), a região da subprefeitura de Pinheiros obteve IFU maior do que quatro dos nove bairros estudados na cidade de Piracicaba/SP (Jardim Monumento, Centro, Cidade Alta e São Dimas).

\section{CONCLUSÕES}

Com a aplicação dos métodos desenvolvidos nesse trabalho foi possível realizar análises quantitativas da arborização urbana da área de estudo. O uso de ferramentas de geoprocessamento e sensoriamento remoto permitiram obter dados físicos do tecido urbano da cidade de São Paulo, sendo o algoritimo Stepwise Linear o que apresentou melhores resultados. Entretanto o tratamento e o processamento dos dados de entrada são de extrema importância para garantir resultados compatíveis com a realidade de campo, além da confecção de um treinamento de boa qualidade para a classificação automática supervisionada.

A subprefeitura de Pinheiros apresentou melhor Índice de Floresta Urbana de arborização urbana, principalmente quando comparada com as regiões das subprefeituras da Mooca e da Sé, sendo que este índice demonstrou ser de grande utilidade para se analisar a relação entre elementos permeáveis e elementos impermeáveis presentes numa região e acompanhar suas modificações em diferentes anos.

\section{AGRADECIMENTOS}

Agradecemos à Fundação de Amparo à Pesquisa do Estado de São Paulo (FAPESP) pelo financiamento desta pesquisa.

\section{REFERÊNCIAS}

ALVAREZ, C.E.; PENTEADO, H.M. Corredores verdes urbanos: estudo da viabilidade de conexão das áreas verdes de Vitória. In: ENCONTRO NACIONAL DE ENSINO DE PAISAGISMO EM ESCOLAS DE ARQUITETURA E URBANISMO NO BRASIL, 8, 2006, São Paulo. Anais... Vitória: UFES. p. 1-12. 
BRAGA, R. Região e gestão metropolitana no final do século XX: uma análise do caso paulista (limitações e avanços). In: SIMPÓSIO NACIONAL DE GEOGRAFIA URBANA, 6 , 1999, PRESIDENTE PRUDENTE. Anais... Presidente Prudente: AGB, 1999. p. 320-325.

BOLAFFI, G. Urban planning in Brazil: past experience, current trends. Habitat International, Oxford, v. 16, n. 2, p. 99-111, 1992.

CENTENO, J.A.S.; RIBEIRO, S.R.A.R. Um método simplificado de fusão de imagens. In: SIMPÓSIO BRASILEIRO DE SENSORIAMENTO REMOTO, 13, 2007, Florianópolis. Anais... São José dos Campos: INPE, 2007. p. 5667-5673.

CIDADE DE SÃO PAULO. Disponível em:

<http://www.cidadedesaopaulo.com/dados.asp>. Acesso em: 05 mar. 2008

INSTITUTO BRASILEIRO DE GEOGRAFIA E ESTATíSTICA, IBGE. Disponível em: <http://www.ibge.gov.br/home/default.php>. Acesso em: 05 mar. 2008.

LANDIS, J.R.; KOCH, G.G. The measurement of observer agreement for categorical data. Biometrics, Washington v. 33, n.1, p. 159-174, 1977.

LILLESAND, T.M.; KIEFER, R.W.; CHIPMAN, J.W. Remote sensing and image interpretation. 5th ed. New York: John Wiley, 2004. $763 \mathrm{p}$.

MICROIMAGES, TNTMIPS: Image Classification. Linconl: Microlmages, 2006. 36p.

MOREIRA, M.A. Fundamentos do sensoriamento remoto e metodologias de aplicação. 3 ed. Viçosa: UFV, 2005. 320 p.

OUMA, Y.O.; TATEISHI, R. Urban-trees extraction from Quickbird imagery using multiscale spectex-filtering and non-parametric classification. Photogrammetric Engineering and Remote Sensing, Washington, v. 63, n. 3, p. 333-351, 2008.

PILOTTO, J. Rede verde urbana: um instrumento de gestão ecológica. 2003. 206 p. Tese (Doutorado em Engenharia de Produção) - Universidade Federal de Santa Catarina, Florianópolis, 2003.

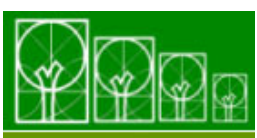

$\mathbf{S} \cdot \mathbf{B} \cdot \mathbf{A} \cdot \mathbf{U}$ Soc. Bras. de Arborização Urbana

REVSBAU, Piracicaba - SP, v.7, n.1, p. 159-181, 2012 
PINHO, C.M.D.; RENNÓ, C.D.; KUX, H.J.H. Avaliação de técnicas de fusão aplicadas à imagem Quickbird. In: SIMPÓSIO BRASILEIRO DE SENSORIAMENTO REMOTO, 12, 2005, Goiânia. Anais... São José dos Campos: INPE, 2005. p. 4225-4232.

PORTAL DA PREFEITURA DA CIDADE DE SÃO PAULO. Disponível em: <http://www.capital.sp.gov.br/portalpmsp/homec.jsp>. Acesso em: 28 jan 2009.

QUICKBIRD. Disponível em <http://www.sat.cnpm.embrapa.br/satelite/quickbird.html>. Acesso em: 20 jun. 2008.

RIBEIRO, S.R.A.; CENTENO, J.A.S. Classificação do uso do solo utilizando redes neurais e o algoritmo MAXVER. In: SIMPÓSIO BRASILEIRO DE SENSORIAMENTO REMOTO, 10, 2001. Foz do Iguaçu. Anais... São José dos Campos: INPE, 2001. p.1341-1348.

SILVA FILHO, D.F. Aplicação de videografia aérea multiespectral na avaliação de floresta urbana. 2004. 88 p. Tese (Doutorado em Agronomia), Faculdade de Ciências Agrárias e Veterinárias, Universidade Estadual Paulista "Júlio de Mesquita Filho", Jaboticabal, 2004.

SILVA FILHO, D.F.; PIVETTA, K.F.L.; COUTO, H.T.Z.; POLIZEL, J.L. Indicadores de floresta urbana a partir de imagens aéreas multiespectrais de alta resolução. Scientia Forestalis, Piracicaba, 67, p.88-100, 2005.

VALERIO, A.M.; SILVA, G.B.S.S.; KAMPEL, M.; STECH, J.L. Avaliação da classificação não supervisionada do uso do solo e tipos de água no reservatório de manso, MT. Geografia: Ensino e Pesquisa, Santa Maria, v. 12, n. 1, p. 4081-4095, 2008.

VRABEL, J. Multispectral imagery band sharpening study. Photogrammetric Engineering and Remote Sensing, Washington, v. 62, n. 9, p. 1075-1083, 1996.

WEBER, C; PETROPOULOU, C.; HIRSCH, J. Urban development in the Athens metropolitan area using remote sensing data with supervised analysis and GIS. International Journal of Remote Sensing, Amsterdan, v. 26, n. 4, p. 785-796, 2005. 